\title{
NOW HEAR THIS! Take action to protect your hearing
}
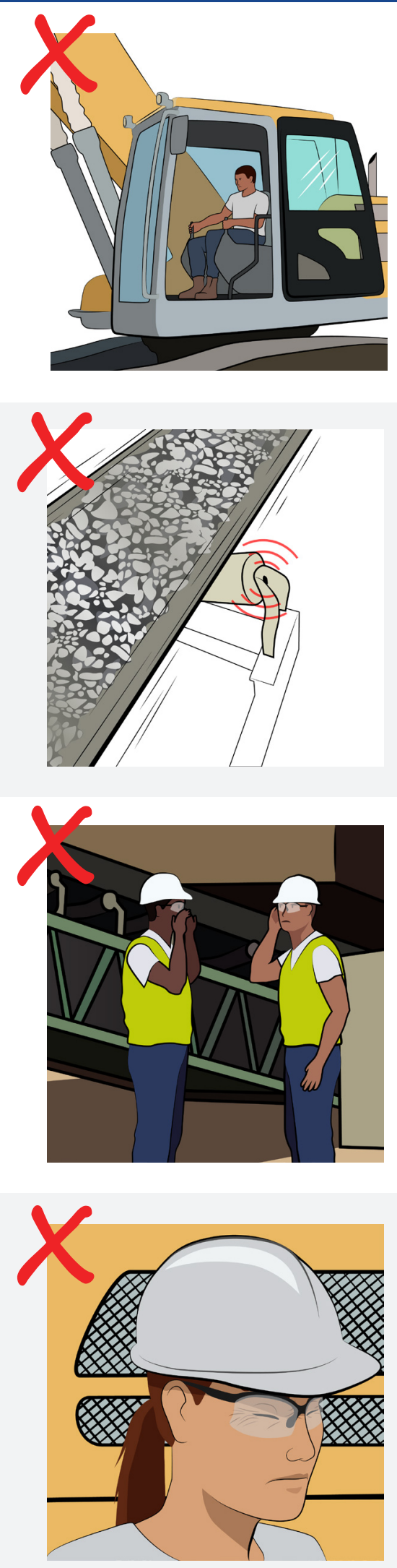

\section{Use Hearing Protection}

- Use hearing protection consistently when working in noise

- Ensure protection is correctly worn with other safety equipment and is comfortable for extended wear

- Avoid circumstances where communication is affected by noise

- Take scheduled breaks and lunch breaks in quiet areas

\section{Use Available Noise Controls}

- Remain inside cabs or other enclosures with the windows and doors closed

Replace noise controls (e.g., curtains, seals) after equipment maintenance or inspection as needed

\section{Maintain Equipment}

- Ensure correct machine lubrication, belt and bolt tension, and motor

- Replace broken, worn out, and misused parts that contribute to equipment noise
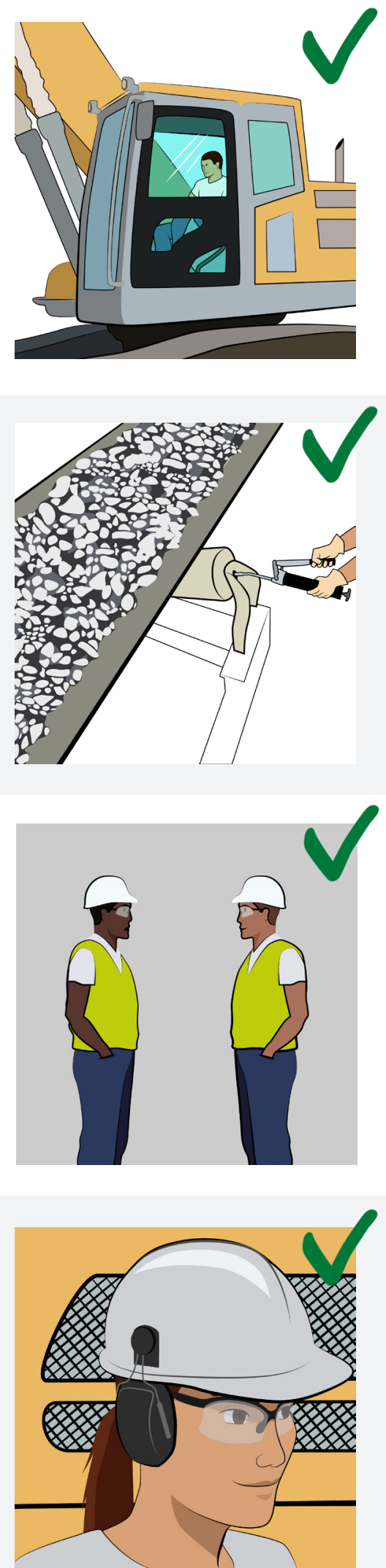

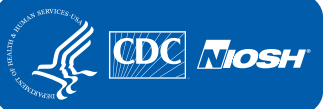

